\title{
EXPERIENCES WITH EARLY CONTACT LENSES AND SOME OF THEIR LATER DEVELOPMENTS*
}

\author{
BY \\ F. A. WILLIAMSON-NOBLE \\ London
}

ConTACr lenses have interested me for a long time. A few years after the first world war I obtained some blown ones from Müller of Wiesbaden and managed to insert one under my eyelids. I did not know at that time about filling the lens with some saline and the visual and cosmetic results were such that they discouraged me from making further experiments.

Zeiss contact lenses became available a few years later and I borrowed a trial set with varying corneal and scleral radii.

The optical effect in these was obtained by variations in the radius of the corneal portion, no lens being ground on them, and one went on trying different lenses to obtain the optimum result. I have no figures regarding toleration, but so far as I remember, this never exceeded a few hours. Two cases remain in my mind. One was that of a patient with 3 or $4 \mathrm{D}$ of astigmatism, in whom fitting the contact lenses was like trying to make half a walnut shell fit on to the back of a spoon, and it was not possible to prevent large bubbles forming inside the lens.

The other was that of a woman who was managing to wear her lenses fairly well. She rang me up one Sunday morning, however, and said she had had her lenses in for some time and that one eye had become foggy, She also mentioned that if she looked at a light she saw haloes round it. I thought that she must have developed glaucoma and asked her to come up and see me. The cornea was obviously hazy and when I removed the contact lens, the epithelium looked oedematous. The ocular tension was normal, however; there was no pain and the cornea cleared a few hours after the lens had been removed. This condition became well known later on through the work of Sattler, and was called Sattler's veil. The aetiology was at first obscure but became clear eventually when it was found that the veil failed to develop if a small ventilating hole was made in the contact lens. This allowed the metabolic products of the cornea to escape and to be replaced by fresh lacrimal fluid thus preventing alterations in its $\mathrm{pH}$.

In the autumn of 1936, before the cause of the veil had been discovered, I heard of the work of Dallos, who was taking moulds of the eyeball, and three of us spent a fortnight in his clinic at Budapest in an attempt to learn his method of fitting contact lenses. We learnt quite a lot, but not enough to enable us

* Received for publication December 21, 1959. 
to obtain satisfactory results, as we discovered when we tried to repeat his methods in a series of evening sessions at Moorfields. We were glad, therefore, when in the Spring of 1937, Dallos came over to England and we were able to install him in London.

The work he has done since then is well known, but some of the recent developments have not yet been published, so that the following cases may be of interest.

\section{Case Reports}

Case 1, a woman aged 49, who developed a cataract in the right eye which I extracted in February, 1951, the left eye being unaffected. The eye took some time to settle down but was eventually fitted with a contact lens with which visual acuity was 6/12. Although binocular vision was obtained, the cosmetic effect was unsatisfactory owing to the highly curved convex surface making the eye appear to bulge forward as compared with its fellow and appear to have a much deeper anterior chamber. We wondered if anything could be done to improve matters and eventually I suggested to Dallos that he should limit the convex lens to the part covering the pupillary area. He has done this, producing a contact lens with a sort of pip, $4 \mathrm{~mm}$. in diameter, over the pupillary area which is quite inconspicuous. Fig. 1 shows what the lens is like and Figs 2 and 3 illustrate the improvement in appearance.

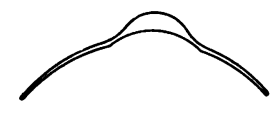

Fig. 1A.-Case 1, contact lens for aphakia as originally made.

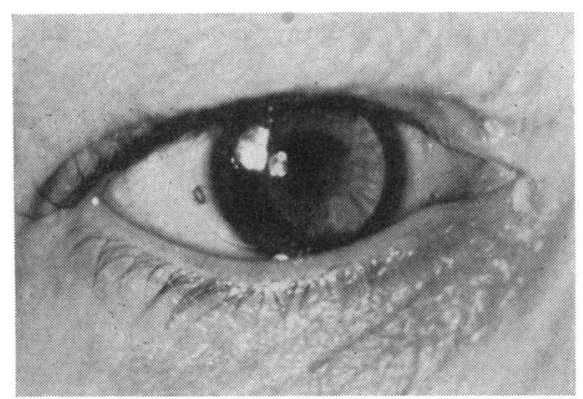

FIG. 2.-Case 1, appearance of original contact lens when worn. The edge of the convex portion is easily seen.

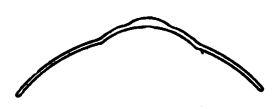

Fig. 1B.-Case 1, contact lens as modified.

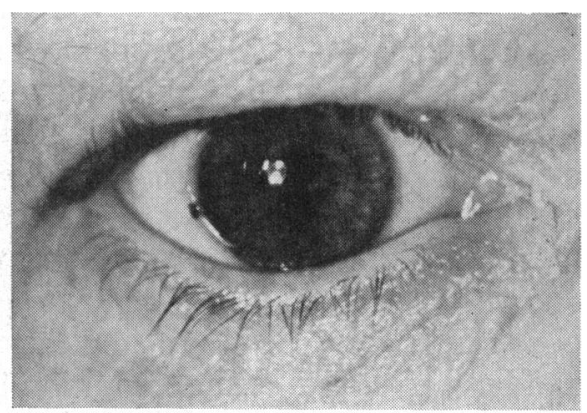

FIG. 3.-Case 1, appearance when wearing modified lens with reduced optical portion.

Case 2, a woman aged 57, was a high myope $-24 \mathrm{D}$ sph. with a $-3 \mathrm{D}$ cyl. in the right eye, and $-22 \mathrm{D}$ sph. in the left, whose lenses were gradually becoming opaque. I extracted her cataracts in 1953. She was restless afterwards and in each eye produced an iris prolapse with resultant astigmatism owing to the presence of a sausage-shaped staphyloma. Her best post-operative visual acuity in 1957 with spectacles was 6/18 in the right eye, with $+3 \mathrm{D}$ cyl., axis $30^{\circ}$, and $6 / 60$ in the left eye, with $+2 \mathrm{D}$ sph., $-13 \mathrm{D}$ cyl., axis $105^{\circ}$. The left eye had diplopia. The staphylomata made the fitting of an ordinary contact lens quite impossible, so at my suggestion Dallos removed a crescentic area of 
glass from the scleral portion of each lens (Fig. 4). The lenses were to be bi-focal, so allowance had to be made for them to slip up and down and the end result was that the patient had a dehiscence so large that it covered about $2 \mathrm{~mm}$. of the upper part of the cornea and left a 2-mm. rim like the handle of a basket in the upper scleral periphery. This kept the contact lens fully balanced and allowed for the lag required for fenestrated bi-focal lenses. She has a satisfactory wearing time with these and the visual acuity is $6 / 12$ partly with the right eye and $6 / 9$ with the left. Her only complaint was that she wanted an intermediate correction, so I have ordered her a pair of $+1.5 \mathrm{D}$ sph. spectacles to wear over the contact lenses.

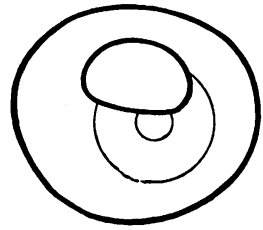

Fig. 4.-Case 2, diagram of contact lens, showing dehiscence above.

An obvious criticism of this case is the staphylomata but there were several reasons why they were not excised. Both cataracts had been extracted intracapsularly, so that there was a definite risk of vitreous loss which might have caused subsequent retinal detachment in eyes with such a high degree of myopia. There was also the fact that the prolapsed iris in each eye was covered by a layer of conjunctiva.

Stenopoeic contact lenses are a recent development which in certain rather desperate cases can be extremely helpful. Their origin goes back quite a long way, some having been blown by Müller of Wiesbaden, possibly betore the beginning of the 20th century. They were made with a white sclera, coloured iris, and clear stenopoeic pupil, with a view to helping albinos on the assumption that the defective pigmentation and light getting through the sclera were responsible for their defective vision.

In fact, most albinos are amblyopic, have nystagmus, and are not helped by increased light contrast.

The manufacture of these lenses was on the lines of blowing artificial eyes. Fig. 5 shows one of Müller's original lenses made at this early date. To improve the optical properties the central portion was blown out of much thicker glass and annealed very carefully so that it could be optically ground and polished.

The lens in Fig. 6 was individually fitted and a very complicated procedure was employed. In this, the plate of glass, pressed over the brass mould, had

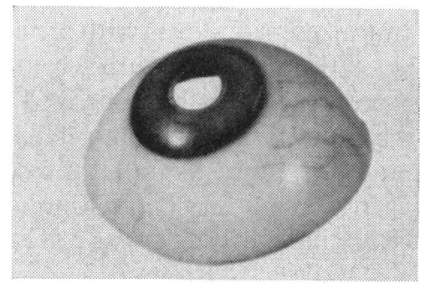

Fig. 5.-One of Müller's original blown contact lenses.

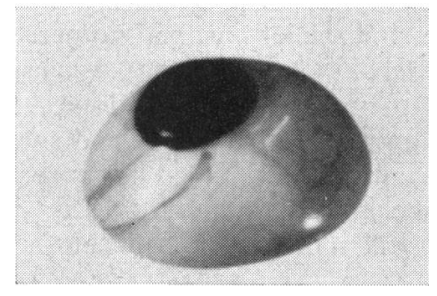

Fig. 6.- One of Müller's lenses which was individually fitted (the cornea appears dark because of the angle of the photograph). 
to include the transparent centre, the opaque inner lining, the white sclera, and the pattern of the iris. There is a case of traumatic aniridia described half a century ago in which intolerable photophobia was relieved by an albino lens. Here of course the opaque sclera was of no importance and it acted as a stenopoeic lens.

This form of lens suggested the extension of stenopoeic lenses to conditions where lack of homogeneity of the media-as opposed to irregularity of the corneal surface-brought about a reduction of visual acuity. Examples of this are nuclear cataract, other slow-growing lens opacities, and various forms of corneal opacities and degenerations.

With regard to the technique of manufacture, if a plastic lens is used, the pigment can be processed into it direct. In glass, an opaque perforated disc is fused into the groove made for it in the lens (Fig. 7A, B).

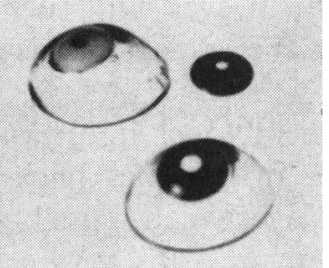

FIG. 7A.-Above left, contact lens with annular area of roughened glass. Right, perforated disc of black opaque glass.

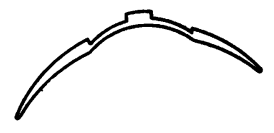

a

Fig. 7B.-Grooved contact lens in section. Stenopoeic disc in section.

The results obtained with these lenses include the following:

Case 3, a man aged 50, was under the care of Mr. A. G. Cross at St. Dunstan's. His right eye had been removed and the left was severely affected by mustard gas keratitis.

In 1942 spectacle lenses would not improve this eye to beyond counting fingers, but with a contact lens he could see 6/24. He was kept under observation until 1953 and then vanished for 5 years. When, at the end of this time, he reappeared, the cornea had become more opaque and vision even with the contact lens could not be improved to beyond 5/60. A stenopoeic lens with a 3- $\mathrm{mm}$. hole placed $1 \mathrm{~mm}$. from the centre at an angle of $15^{\circ}$ from the optical axis of the eye, gave him 6/24. He could wear this lens with comfort and was able to get about without the escort he had formerly required.

Case 4, a woman aged 52, was under the care of Mr. J. E. M. Ayoub. She was highly myopic, and the left eye had no perception of light, but in the right eye, with an ordinary contact lens, the visual acuity was 6/24. A year later, without Mr. Ayoub's knowledge, she had an optical iridectomy done. The visual acuity could not then be improved beyond counting fingers at 2 metres and she had troublesome diplopia. A contact lens with a 3-mm. central hole enabled her to see $6 / 18$, however, and although she hated the cosmetic effect, she wore it all day. An attempt was made to improve matters by putting a replica of iris pigment on the lens, but this made the eye even more unsightly and was not adopted.

Case 5, a man aged 34, had been treated by Mr. H. Neame for mustard gas keratitis. Those who have not seen these sad cases may be unaware that although in some the condition remains stationary at a certain stage, others seem to be unaffected by treatment, the 
cornea becoming more and more opaque and thinned, until finally it ruptures and the eye is lost. A patient of mine, in whom one eye was badly affected, first knew of it when it ruptured while he was playing tennis. He refused to have the eyeball removed and it became infected. This was before the days of antibiotics and the infection travelled up to the brain, causing a fatal meningitis.

Case 5 was not so bad as this, but the history is certainly pathetic. He was fitted with contact lenses in both eyes in 1938 and the visual acuity in the right eye came up to 6/12, and that in the left to $6 / 6$. In 1947 , however, the acuity had decreased to $6 / 18$ and $6 / 24$, and in February 1951, to $6 / 24$ and 6/60; 9 months later, the right eye still saw $6 / 24$ but the left only $1 / 60 ; 3$ years after this, the right eye was hopeless because the cornea was quite degenerate, but the left eye, which with an ordinary contact lens could not see even $6 / 60$, with a stenopoeic one saw $6 / 24$. The patient has retained this degree of vision, wears his lens all day, and sees with it enough to play golf.

Stenopoeic contact lenses are also sometimes of use in cases of cataract, particularly when the opacity is localized, as in the nuclear type.

Case 6 was one of Mr. A. Lister's patients. This man's left eye had no perception of light and the right eye had a nuclear cataract. The visual acuity in this eye was $4 / 60$ with correction, and with an ordinary contact lens, much the same. With a central stenopoeic, however, it could be improved to $6 / 18$, and he wore this lens for 3 years, after which he was not seen.

Case 7, a boy aged 16, was in the care of Sir Stewart Duke-Elder. His left eye was amblyopic and practically useless and his right eye received an injury while he was fencing. As a result, the cornea was distorted and there was a scar in the sclera. An ordinary contact lens improved his vision to $6 / 18$, but with a central stenopoeic hole he could see 6/9. He used this lens when he was studying, but preferred the other for use out of doors.

At first sight it may seem rather odd that a patient should prefer $6 / 18$ to $6 / 9$, but the reason is that a stenopoeic hole reduces the field of vision especially on the temporal side. Exact figures cannot be quoted for this because the field reduction depends upon various factors:

(1) The depth of the anterior chamber and so the distance of the stenopoeic hole from the main plane of the eye.

(2) The diameter of the hole.

(3) The position of the hole, in one case, for example, the hole had to be $1 \mathrm{~mm}$. nasal to the centre of the cornea, thereby cutting out comparatively more of the temporal field.

(4) High degrees of anomaly of refraction, e.g. aphakia and high myopia, will influence the amount of field which is lost.

These cases would seem to show that, although nowadays corneal or microlenses are being widely used, especially in America, there is still a place for the fully-fitted type.

I should like to thank Dr. J. Dallos for his co-operation in the preparation of this paper, the ophthalmic surgeons who have kindly allowed me to refer to their cases, and the Institute of Ophthalmology for providing the illustrations. 